\title{
Vitamin D deficiency is associated with disease activity in patients with Crohn's disease
}

\author{
Kyoung Ho Ko, You Sun Kim, Bo Kyung Lee, Jong Hyun Choi, Yong Moon Woo, Jin Young Kim, \\ Jeong Seop Moon \\ Department of Internal Medicine, Seoul Paik Hospital, Inje University College of Medicine, Seoul, Korea
}

\begin{abstract}
Background/Aims: Previous data suggest that vitamin D has a significant role in inflammatory bowel disease (IBD). We investigated the incidence of vitamin D deficiency in Korean patients with IBD and the correlation between serum vitamin D level and disease activity. Methods: We retrospectively analyzed the medical records of patients with IBD whose serum vitamin D levels were checked. Deficiency of 25 -hydroxyvitamin D was defined as $<20 \mathrm{ng} / \mathrm{mL}$. Disease activity was evaluated using the partial Mayo score for ulcerative colitis ( $\geq 2$ defined as active disease) and Harvey-Bradshaw index for Crohn's disease ( $\geq 4$ defined as active disease). Results: We enrolled 87 patients with IBD (ulcerative colitis [UC], 45; Crohn's disease [CD], 42). Among them, $65.5 \%$ (57/87) were men, with a mean age of $44.9 \pm 15.1$ years (range, $18-75$ years). The mean duration of disease was $4.7 \pm 4.8$ years (range, $0.1-17.1$ years). Vitamin D deficiency was found in $73.6 \%(64 / 87)$ of patients with IBD. Patients with IBD (mean vitamin D level, $16.3 \pm 9.0 \mathrm{ng} / \mathrm{mL}$ ) showed lower vitamin D level than the healthy control group (mean vitamin D level, $20.4 \pm 7.0 \mathrm{ng} / \mathrm{mL})$, with no statistically significant difference $(P=0.136)$. Disease activity was inversely correlated with vitamin D deficiency in patients with $\mathrm{CD}(P=0.007)$. However, no correlation was observed in patients with UC $(P=0.134)$. Conclusions: Approximately $75 \%$ of Korean patients with IBD showed vitamin D deficiency state. Vitamin D deficiency is associated with disease activity, particularly in patients with CD. (Intest Res 2019;17:70-77)
\end{abstract}

Key Words: Inflammatory bowel diseases; Vitamin D deficiency; Disease activity

\section{INTRODUCTION}

Inflammatory bowel disease (IBD), including UC and CD, is a GI disorder with a chronic and recurrent inflammatory process that requires lifelong treatment. IBD is highly prevalent in developed countries, such as North America and Europe, and affects up to $0.5 \%$ of the general population. Despite previous low incidence rates, Asia has experienced a meaningful increase in IBD incidence over the last 20 years, whereas Europe is experiencing a stagnation or decline ${ }^{1,2}$ Patients

Received February 15, 2018. Revised June 6, 2018. Accepted July 9, 2018. Correspondence to You Sun Kim, Department of Internal Medicine, Seoul Paik Hospital, Inje University College of Medicine, 9 Mareunnae-ro, Jung-gu, Seoul 04551, Korea. Tel: +82-2-2270-0012, Fax: +82-2-2270-0257,

E-mail:yousunk69@korea.com

ORCID Kyoung Ho Ko (https://orcid.org/0000-0002-3835-3130), You Sun Kim (https://orcid.org/0000-0002-5156-3458) with IBD may have a variety of symptoms, such as diarrhea with blood and mucus, abdominal pain, and fatigue, that reduce the quality of life. ${ }^{3}$ There is much interest in the nutritional status of patients with IBD, and efforts are needed to improve nutritional status. ${ }^{4-6}$

Vitamin D deficiency is more common in patients with IBD than in the general population. The incidence of vitamin D deficiency in IBD patients ranges from $16 \%$ to $95 \%$, and is known to frequently occur in CD than in UC. ${ }^{7-10}$ Many factors can contribute to vitamin D deficiency, such as malabsorption, reduced exposure to sunlight, lack of physical activity, reduced dietary and vitamin D intake, and smoking. ${ }^{11,12}$ The most stable form of measurement of vitamin D in serum is 25-hydroxyvitamin $\mathrm{D}$ (25-OH vitamin $\mathrm{D})$, and its level reflects the vitamin D provided by sun exposure, dietary intake, supplementation, and storage.

Several epidemiological studies have found a significant 
inverse relationship between vitamin D level and the development of IBD..$^{7-9}$ Although the effects of vitamin D supplementation on the outcome of the disease and the timing of proper vitamin D supply are not yet clear, several studies suggest that vitamin D plays an important role in the course of IBD. ${ }^{11,13,14}$

This study aimed to determine the correlation between serum vitamin D level and disease activity in patients with IBD.

\section{METHODS}

\section{Patients}

Our study was performed in the gastroenterology clinic of Inje University Seoul Paik Hospital for patients diagnosed with UC or CD from January 2000 to December 2017. The diagnosis of UC or CD was made based on the diagnostic guideline suggested by the IBD study group of the Korean Association for the Study of Intestinal Disease. ${ }^{15,16}$ The medical records of the enrolled patients were retrospectively analyzed, including demographic, laboratory, endoscopic, radiological, pathological, and other clinical data. Patients were excluded if they had no data of serum vitamin D level or had comorbidities affecting serum vitamin D levels (i.e., renal failure, liver disease, lactation, pregnancy, medications, such as vitamin D supplements and anticonvulsants). The 80 of normal control group was selected based on age and sex among those had normal colonoscopy finding and serum vitamin D level was measured during health checkup in 2017 at Seoul Paik Hospital of Inje University. Among them, $63.8 \%(51 / 80)$ were men, with a mean age of $40.4 \pm 13.6$ years (range, 29-73 years). The study was conducted after the approval of the Ethics Committee of Seoul Paik Hospital of Inje University (IRB File No. 2017-12-007). Informed consent was waived.

\section{Measurements}

Patients' characteristics including disease duration, location and behavior, IBD-related surgeries, and medical history were obtained from the clinical records. Physical examinations were performed, and height and weight were measured on single calibrated scales. Hemoglobin, white blood cell counts (WBC), CRP, ESR, albumin, and cholesterol levels were also measured as indicators of inflammation and disease severity on the same day vitamin D level was measured. Medication history has been checked for cumulative drugs used since the disease was diagnosed.

Vitamin D status was obtained through measurement of serum 25-OH vitamin D level by using a fully automated immunoassay (ADVIA Centaur XP ${ }^{\circledR}$, Siemens, Munich, Germany) because it was considered as the best measurement of an individual's vitamin D status. ${ }^{10}$

\section{Definitions of Disease Activity and Vitamin D Deficiency}

The primary outcome was the association of vitamin D deficiency with IBD disease activity. Disease activity was assessed using the partial Mayo score and Harvey-Bradshaw index (HBI) for UC and CD, respectively. The partial Mayo score includes reported stool frequency, presence of rectal bleeding, and a global assessment of the physician. The HBI includes general well-being, number of liquid stools per day, abdominal pain, abdominal mass, and complications. For UC, a partial Mayo score of 2 or higher was defined as active disease. In CD, an HBI score of $<4$ was defined as clinical remission, and a score of 4 or higher was defined as active disease. ${ }^{17-21}$ The patients were classified based on their serum $25-\mathrm{OH}$ vitamin D levels. Vitamin D deficiency was defined as vitamin D level of $<20 \mathrm{ng} / \mathrm{mL}^{22}$

\section{Statistical Analysis}

Data were analyzed using the SPSS software version 24.0 (IBM Corp., Armonk, NY, USA). Consecutive variables were summarized using mean and standard deviation, and categorical variables were represented by the corresponding numbers and ratios. We used independent sample $t$-test to compare quantitative data. Correlations between variables were verified by the Fisher exact test. Binary logistic regression, for disease activity and vitamin D level, was adjusted considering independent variables, such as age, sex, BMI, smoking, disease duration, and laboratory variables (serum vitamin D, hemoglobin, WBC, ESR, CRP, and albumin). A $P$ value $<0.05$ was considered statistically significant.

\section{RESULTS}

\section{Baseline Characteristics}

We enrolled 87 patients with IBD in this study (UC, 45; CD, 42). Among them, $65.5 \%$ were men, with a mean age of $44.9 \pm 15.1$ years (range, $18-75$ years). The mean duration of the disease was $4.7 \pm 4.8$ years (range, $0.1-17.1$ years). 5-ASA, prednisolone, immunomodulators, and anti-TNF therapy were administered in $98.9 \%, 56.3 \%, 56.3 \%$, and $32.2 \%$ of patients, respectively. Five patients $(5.7 \%)$ underwent small bowel resection and colectomy for complications, such as stenosis, recurrent hemorrhage, and abscess. Table 1 shows the 
demographic data and clinical characteristics of the patients.

\section{Vitamin D Levels}

Vitamin D deficiency $(<20 \mathrm{ng} / \mathrm{mL})$ was found in $73.6 \%$ of patients with IBD (UC, 73.3\%; CD, 73.8\%). Patients with IBD showed a lower mean of vitamin D level $(16.3 \pm 9.0 \mathrm{ng} / \mathrm{mL})$ than the healthy control group $(20.4 \pm 7.0 \mathrm{ng} / \mathrm{mL})$, with no statistically significant difference $(P=0.136)$ (Fig. 1$)$.

The mean vitamin $\mathrm{D}$ level in patients with $\mathrm{CD}$ was lower than that in those with UC, which was not statistically significant (15.4 $\pm 8.2 \mathrm{ng} / \mathrm{mL}$ vs. $17.1 \pm 9.7 \mathrm{ng} / \mathrm{mL}, P=0.640)$ (Fig. 1 ).
In the univariate analysis, the age of patients in the vitamin D deficiency group was significantly younger $(41.3 \pm 15.8$ years) than that of those in the normal group (52.6 \pm 10.6 years) $(P=0.011)$, but sex, BMI, duration of disease, disease location, drug use, and surgery were not significantly associated with serum vitamin D level. Also, the presence of anemia, leukocytosis, higher ESR and CRP levels, and lower albumin and total cholesterol levels were not significantly associated with vitamin D levels. Meanwhile, in the binary logistic regression analysis, age and disease activity were independently associated with vitamin D levels and the ORs

Table 1. Demographic Data and Clinical Characteristics of 87 Patients with IBD

\begin{tabular}{|c|c|c|c|}
\hline Characteristics & $\begin{array}{c}\text { UC } \\
(n=45)\end{array}$ & $\begin{array}{c}C D \\
(n=42)\end{array}$ & $\begin{array}{l}\text { All patients } \\
\quad(\mathrm{n}=87)\end{array}$ \\
\hline Age (yr) & $48.5 \pm 13.7$ & $40.9 \pm 15.6$ & $44.9 \pm 15.1$ \\
\hline Male sex & $28(62.2)$ & $29(69.0)$ & $57(65.5)$ \\
\hline BMI $\left(\mathrm{kg} / \mathrm{m}^{2}\right)$ & $22.6 \pm 3.4$ & $21.5 \pm 4.5$ & $22.1 \pm 4.0$ \\
\hline Smoker & $7(15.6)$ & $7(16.7)$ & $14(16.1)$ \\
\hline Disease duration (yr) & $6.4 \pm 5.7$ & $2.9 \pm 2.4$ & $4.7 \pm 4.8$ \\
\hline Disease location for UC & & - & - \\
\hline Proctitis & $15(33.3)$ & & \\
\hline Left-sided colitis & $9(20.0)$ & & \\
\hline Extensive colitis & $21(46.7)$ & & \\
\hline Disease location for $C D$ & - & & - \\
\hline Ileal & & $11(26.2)$ & \\
\hline Colonic & & $8(19.0)$ & \\
\hline Ileocolonic & & $23(54.8)$ & \\
\hline \multicolumn{4}{|l|}{ Medication } \\
\hline 5-ASA & $45(100)$ & $41(97.6)$ & $86(98.9)$ \\
\hline Prednisolone & $25(55.6)$ & $24(57.1)$ & $49(56.3)$ \\
\hline Immunomodulators (AZA/MTX/6-MP) & $23(51.1)$ & $26(61.9)$ & $49(56.3)$ \\
\hline Anti-TNF (infliximab/adalimumab) & $11(24.4)$ & $17(40.5)$ & $28(32.2)$ \\
\hline IBD-related surgery & $2(4.4)$ & $3(7.1)$ & $5(5.7)$ \\
\hline Mean 25-OH vitamin D (ng/mL) & $17.1 \pm 9.7$ & $15.4 \pm 8.2$ & $16.3 \pm 9.0$ \\
\hline Vitamin D deficiency (<20 ng/mL) & $33(73.3)$ & 31 (73.8) & 64 (73.6) \\
\hline Hemoglobin (g/dL) & $13.5 \pm 2.3$ & $13.9 \pm 1.5$ & $13.7 \pm 1.9$ \\
\hline WBC $\left(\times 10^{6}\right.$ cells $\left./ \mu \mathrm{L}\right)$ & $7.4 \pm 2.8$ & $6.7 \pm 2.8$ & $7.0 \pm 2.8$ \\
\hline ESR $(\mathrm{mm} / \mathrm{hr})$ & $21.6 \pm 23.9$ & $27.7 \pm 23.8$ & $24.6 \pm 23.9$ \\
\hline $\mathrm{CRP}(\mathrm{mg} / \mathrm{dL})$ & $0.9 \pm 2.5$ & $0.4 \pm 0.5$ & $0.7 \pm 1.9$ \\
\hline Albumin (g/dL) & $4.3 \pm 0.4$ & $4.3 \pm 0.6$ & $4.3 \pm 0.5$ \\
\hline Total cholesterol (mg/dL) & $181.3 \pm 35.0$ & $173.6 \pm 43.0$ & $177.6 \pm 39.0$ \\
\hline
\end{tabular}

Values are presented as mean \pm SD or number $(\%)$.

5-ASA, 5-aminosalicylic acid; AZA, azathioprine; MTX, methotrexate; 6-MP, 6-mercaptopurine; 25-OH vitamin D, 25-hydroxyvitamin D; WBC, white blood cells. 
were $0.96(P=0.023 ; 95 \% \mathrm{CI}, 0.92-0.99)$ and $5.06(P=0.044$; 95\% CI, 1.04-7.59) (Table 2).

\section{Vitamin $D$ and Disease Activity}

Among the enrolled patients with IBD, 27 (31.0\%) and 60 patients $(69.0 \%)$ had active disease and were in remission, respectively. Compared with patients in remission, those with active disease had more frequent use of steroid and immunomodulators ( $74.1 \%$ vs. $48.3 \%, P=0.035$ ), as well as biological therapy (55.6\% vs. $21.7 \%, P=0.003)$, lower se-

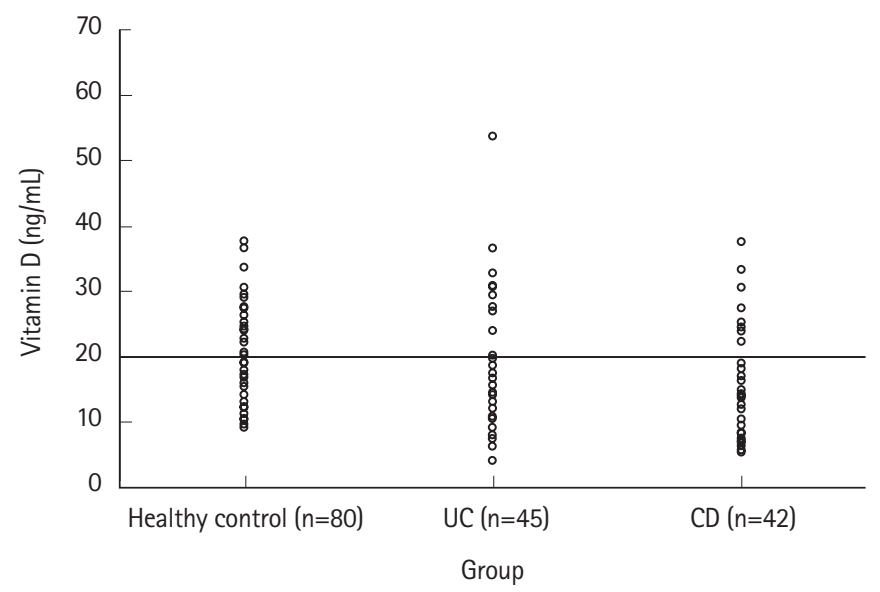

Fig. 1. Comparison of vitamin D levels between healthy control group, UC and CD patients. rum vitamin D level $(11.4 \pm 6.3 \mathrm{ng} / \mathrm{mL}$ vs. $18.1 \pm 9.2 \mathrm{ng} / \mathrm{mL}$, $P=0.012$ ), and more frequent vitamin D deficiency (95.8\% vs. $65.1 \%, P=0.003$ ) in the univariate analysis. With regard to serological biomarkers, patients with active disease were found to have higher WBC count, ESR, and CRP levels than those who were in clinical remission. However, in the binary logistic regression analysis, only low levels of vitamin D were independently associated with disease activity, with an OR of 0.91 ( $P=0.028 ; 95 \% \mathrm{CI}, 0.84-0.99)$.

On subgroup analysis, only patients with CD with active disease had lower serum vitamin D level $(9.6 \pm 2.9 \mathrm{ng} / \mathrm{mL}$ vs. $18.3 \pm 8.5 \mathrm{ng} / \mathrm{mL}, P=0.001)$ and more frequent vitamin $\mathrm{D}$ deficiency ( $100 \%$ vs. $60.7 \%, P=0.007)$ than patients in clinical remission, which was statistically significant (Table 3 ).

Disease activity was inversely correlated with vitamin D deficiency in patients with $\mathrm{CD}(P=0.007)$. However, no correlation was observed in patients with UC $(P=0.134)$ (Fig. 2).

\section{DISCUSSION}

The sources of vitamin D are the synthesis of the skin through sunlight and the intake of food or supplement containing vitamin D. Although the main cause is still unclear, patients with IBD have a higher incidence of vitamin D deficiency than the general population. ${ }^{23}$ In this study, we identified the same results as several previous studies that

Table 2. Multivariate Analysis of Vitamin D Status and Selected Variables in IBD

\begin{tabular}{|c|c|c|c|c|}
\hline Variable & $\begin{array}{c}\text { Normal } \\
(\geq 20 \mathrm{ng} / \mathrm{mL}) \\
(n=23)^{\mathrm{a}}\end{array}$ & $\begin{array}{c}\text { Deficiency } \\
(<20 \mathrm{ng} / \mathrm{mL}) \\
(n=64)^{\mathrm{b}}\end{array}$ & OR $(95 \% \mathrm{Cl})$ & $P$-value \\
\hline Male sex & $14(60.9)$ & $43(67.2)$ & $0.75(0.19-3.01)$ & 0.680 \\
\hline BMI $\left(\mathrm{kg} / \mathrm{m}^{2}\right)$ & $22.8 \pm 3.4$ & $21.8 \pm 4.2$ & $1.03(0.86-1.24)$ & 0.727 \\
\hline Active disease & $2(8.7)$ & $26(40.6)$ & $5.06(1.04-7.59)$ & $<0.050$ \\
\hline Disease duration (yr) & $5.4 \pm 5.4$ & $4.5 \pm 4.5$ & $0.99(0.86-1.15)$ & 0.971 \\
\hline Hemoglobin (g/dL) & $13.9 \pm 1.8$ & $13.6 \pm 2.0$ & $0.93(0.63-1.35)$ & 0.688 \\
\hline WBC $\left(\times 10^{6}\right.$ cells $\left./ \mu \mathrm{L}\right)$ & $6.4 \pm 2.4$ & $7.3 \pm 2.9$ & $1.00(1.00-1.00)$ & 0.828 \\
\hline Total cholesterol (mg/dL) & $181.7 \pm 27.0$ & $176.1 \pm 42.6$ & $1.00(0.99-1.02)$ & 0.985 \\
\hline
\end{tabular}

Values are presented as mean \pm SD or number (\%).

${ }^{a} U C(n=12)$ and $C D(n=11)$.

${ }^{b} U C(n=33)$ and $C D(n=31)$.

WBC, white blood cells. 
Table 3. Subgroup Analysis between Patients in Remission and Patients with Active Disease

\begin{tabular}{|c|c|c|c|c|c|c|}
\hline \multirow[b]{2}{*}{ Variable } & \multicolumn{3}{|c|}{ UC $(n=45)$} & \multicolumn{3}{|c|}{$C D(n=42)$} \\
\hline & $\begin{array}{l}\text { Active disease } \\
\quad(n=13)\end{array}$ & $\begin{array}{l}\text { In remission } \\
\quad(n=32)\end{array}$ & $P$-value & $\begin{array}{l}\text { Active disease } \\
\quad(n=14)\end{array}$ & $\begin{array}{c}\text { In remission } \\
\quad(n=28)\end{array}$ & $P$-value \\
\hline Disease duration (yr) & $3.9 \pm 4.9$ & $7.4 \pm 5.8$ & $0.282^{\mathrm{a}}$ & $2.8 \pm 2.7$ & $3.0 \pm 2.3$ & $0.679^{a}$ \\
\hline \multicolumn{7}{|l|}{ Medication } \\
\hline 5-ASA & $13(100)$ & $32(100)$ & $>0.999^{b}$ & $13(92.9)$ & $28(100)$ & $0.333^{b}$ \\
\hline Prednisolone & $8(61.5)$ & $17(53.1)$ & $0.745^{b}$ & $12(85.7)$ & $12(42.9)$ & $<0.050$ \\
\hline Immunomoulators (AZA/MTX/6-MP) & $9(69.2)$ & $14(43.8)$ & $0.189^{b}$ & $11(78.6)$ & $15(53.6)$ & $0.180^{b}$ \\
\hline Anti-TNF (infliximab/adalimumab) & $7(53.8)$ & $4(12.5)$ & $<0.050$ & $8(57.1)$ & $9(32.1)$ & $0.184^{b}$ \\
\hline IBD-related surgery & $1(7.7)$ & $1(3.1)$ & $0.499^{b}$ & $2(14.3)$ & $1(3.6)$ & $0.254^{b}$ \\
\hline Mean $25-\mathrm{OH}$ vitamin $\mathrm{D}(\mathrm{ng} / \mathrm{mL})$ & $13.0 \pm 8.4$ & $18.7 \pm 9.9$ & $0.361^{a}$ & $9.6 \pm 2.9$ & $18.3 \pm 8.5$ & $<0.050$ \\
\hline Vitamin D deficiency $(<20 \mathrm{ng} / \mathrm{mL})$ & $12(92.3)$ & $21(65.6)$ & $0.134^{b}$ & $14(100)$ & $17(60.7)$ & $<0.050$ \\
\hline Hemoglobin $(g / d L)$ & $14.1 \pm 1.9$ & $13.3 \pm 2.4$ & $0.329^{a}$ & $13.4 \pm 1.9$ & $14.1 \pm 1.2$ & $0.051^{\mathrm{a}}$ \\
\hline WBC $\left(\times 10^{6}\right.$ cells $\left./ \mu \mathrm{L}\right)$ & $8.0 \pm 3.0$ & $7.1 \pm 2.7$ & $0.545^{\mathrm{a}}$ & $7.5 \pm 4.1$ & $6.2 \pm 1.8$ & $<0.050$ \\
\hline ESR $(\mathrm{mm} / \mathrm{hr})$ & $32.2 \pm 31.3$ & $17.3 \pm 19.1$ & $0.169^{a}$ & $33.9 \pm 32.4$ & $24.6 \pm 18.0$ & $<0.050$ \\
\hline $\mathrm{CRP}(\mathrm{mg} / \mathrm{dL})$ & $2.2 \pm 4.4$ & $0.4 \pm 0.6$ & $<0.050$ & $0.6 \pm 0.5$ & $0.4 \pm 0.5$ & $0.503^{\mathrm{a}}$ \\
\hline Albumin (g/dL) & $4.3 \pm 0.3$ & $4.3 \pm 0.4$ & $0.989^{a}$ & $4.1 \pm 0.9$ & $4.4 \pm 0.2$ & $<0.050$ \\
\hline Total cholesterol (mg/dL) & $176.2 \pm 23.5$ & $183.4 \pm 38.8$ & $0.078^{\mathrm{a}}$ & $168.6 \pm 61.3$ & $176.1 \pm 31.3$ & $0.120^{\mathrm{a}}$ \\
\hline
\end{tabular}

Values are presented as mean \pm SD or number (\%).

${ }^{a}$ Independent sample $t$-test.

${ }^{b}$ Fisher exact test.

5-ASA, 5-aminosalicylic acid; AZA, azathioprine; MTX, methotrexate; 6-MP, 6-mercaptopurine; 25-OH vitamin D, 25-hydroxyvitamin D; WBC, white blood cells.
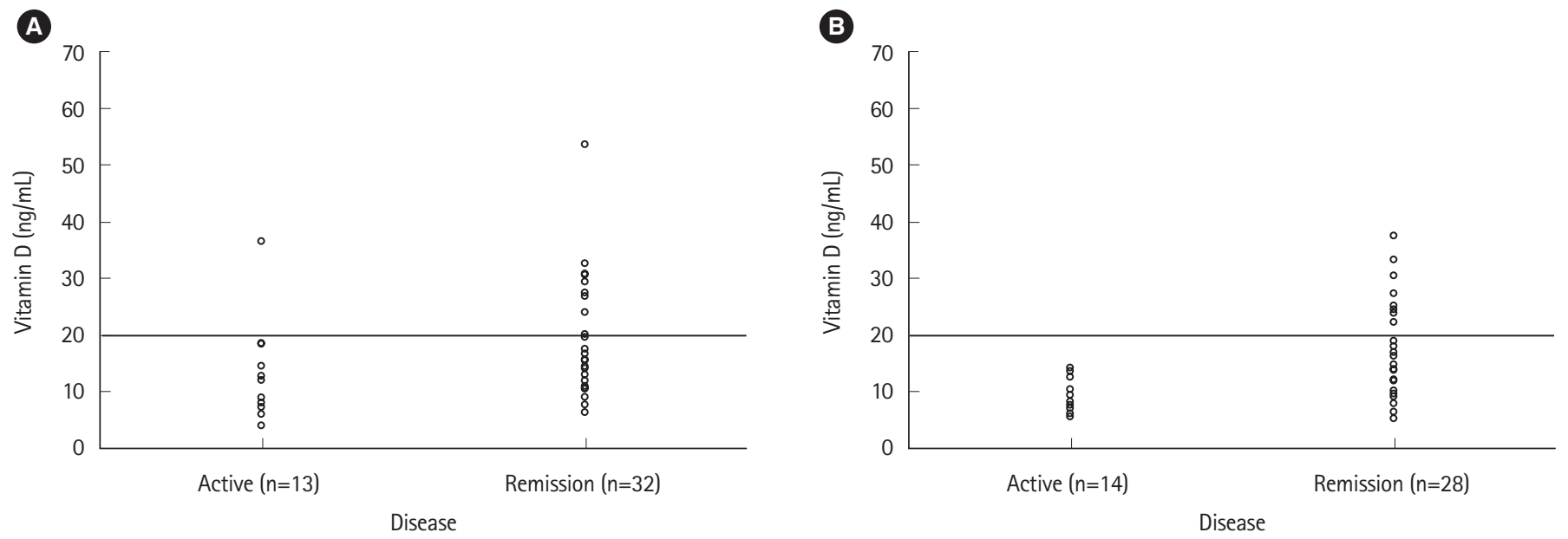

Fig. 2. Association between vitamin $D$ levels and disease activity in patients with IBD. $(A) \cup C(n=45)$ and $(B) C D(n=42)$.

reported a higher vitamin D deficiency incidence in IBD. The incidence of vitamin D deficiency in patients with IBD in our study (73.6\%) was relatively higher than that reported in a previous retrospective American study $(49.8 \%),{ }^{9}$ but lower than that in the recent study conducted in Korea
(89.2\%). ${ }^{24}$ These results are consistent with previous findings that Asian people tended have a higher incidence of vitamin D deficiency than Caucasians. ${ }^{25}$ In addition, differences in research results may be due to other criteria for defining low vitamin D levels and environmental factors, 
and patient characteristics such as demographics, physical activity, and nutritional status. In our study, we considered albumin and cholesterol levels as indicators of nutritional status and investigated the association with vitamin D deficiency. However, we could not find a significant relationship between albumin and cholesterol level and vitamin D level, so we assumed that vitamin D deficiency was not caused by malnutrition.

There are several causes for vitamin D deficiency. Some studies show that lack of exposure to sunlight can be an important cause of vitamin D deficiency in patients with IBD, particularly from northern climates. They demonstrated that an association was found between winter season and vitamin D deficiency due to low sunlight and UV-B exposure. ${ }^{26}$ Inadequate physical activity in patients with chronic illness, known as another risk factor associated with vitamin D deficiency. In addition, bowel resection is found to be associated with vitamin D deficiency owing to the discontinuation of the enterohepatic circulation in the terminal ileum, interrupting the absorption of fat-soluble vitamins, such as vitamin D. ${ }^{27,28}$ However, those studies have not persistently investigated the effect of bowel resection on vitamin D deficiency, and our results also did not find a significant correlation between bowel resection and vitamin $\mathrm{D}$ deficiency $(P=0.319)$. In addition, vitamin D levels and location of IBD lesions are not significantly related in this study $(P=0.463)$.

Few reports have evaluated the effect of vitamin D supplementation on the clinical course of IBD in clinical trials. A recent American study investigating 138 patients showed that patients who did not receive vitamin $\mathrm{D}$ supplementation had lower levels of vitamin D and more frequent use of the health care system. ${ }^{29}$

In some other studies, inflammatory activity of CD has been found to be associated with vitamin D deficiency. Some researchers claimed that low vitamin D levels are associated with active disease, ${ }^{29,30}$ whereas others do not. ${ }^{31}$ Finding this association is complicated because of the many variables in assessing disease activity.

In contrast, studies on disease activity in UC and vitamin D deficiency are insufficient, with conflicting results. Although our study and a Romanian study observed that no association was found between vitamin D deficiency and disease activity, an American study of 34 patients with UC showed that they were associated. ${ }^{32,33}$ In addition, in a recent cohort study of 368 patients with UC, patients with normal vitamin D levels had significantly lower disease activity, but no association was found between systemic markers of inflammation and vitamin D levels. ${ }^{29}$
This study found an inverse correlation between low vitamin D levels and disease activity in patients with CD. We observed that patients with CD with low vitamin D levels had significantly higher disease activity measured by HBI. However, such association was not observed in patients with UC. These results were consistent with a large retrospective study of 200 outpatients with IBD at a Norwegian research institution (UC, 78; CD, 122). ${ }^{34}$

Other variables, particularly ESR, CRP, and albumin levels, were associated with clinical disease activity, but no significant association was found between serum vitamin D status and inflammatory markers (ESR, CRP, and leukocytes) in our study. These results can be supported by the theory that serum vitamin D levels can reflect local inflammation in tissues rather than systemic inflammation, but further studies are needed to clarify the relationship. ${ }^{30}$ Meanwhile, a recent study evaluated fecal calprotectin, which showed a significant inverse correlation with vitamin D levels. ${ }^{30,35}$

Our study has one interesting finding. The risk of vitamin D deficiency is generally high in the elderly, but in our study, vitamin D deficiency was significantly higher in the younger age. A recent American cohort study of patients with IBD also found the similar results. ${ }^{29}$ These findings may have been presumed to be caused by intake of vitamin Dcontaining nutrients without prescription or by differences in lifestyle and eating habits.

Our investigation has some limitations. First, this study was retrospective and performed on a limited number of patients at a single outpatient clinic; hence, the results were difficult to generalize. In addition, previously limited screening conditions and cost problems have not led to the examination of intestinal inflammatory markers, such as fecal calprotectin in many patients with IBD, which can provide better information on disease activity. Furthermore, we did not evaluate the influence of vitamin D supplementation on disease activity, patient symptoms, quality of life, and treatment changes.

In conclusion, vitamin D deficiency is frequently found in patients with IBD. In addition, vitamin D deficiency is associated with increased disease activity in patients with CD. However, no significant association between vitamin D levels and serum inflammatory markers was found. Because of the high rate of vitamin D deficiency in patients with IBD, appropriate vitamin D screening and supplementation with proper amounts of vitamin D may be helpful. More large prospective studies and clinical trials are needed to clarify the role of vitamin $\mathrm{D}$ in the clinical course of patients with IBD and to recognize its importance. 


\section{FINANCIAL SUPPORT}

The authors received no financial support for the research, authorship, and/or publication of this article.

\section{CONFLICT OF INTEREST}

No potential conflict of interest relevant to this article was reported.

\section{AUTHOR CONTRIBUTION}

Conceptualization: Kim YS, Moon JS, Ko KH, and Lee BK. Methodology: Kim YS. Formal analysis: Kim YS, Ko KH, Choi JH, Woo YM, and Kim JY. Project administration: Kim YS. Visualization: Kim YS. Writing-original draft: Ko KH. Writingreview and editing: Kim YS. Approval of final manuscript: All authors.

\section{REFERENCES}

1. Molodecky NA, Soon IS, Rabi DM, et al. Increasing incidence and prevalence of the inflammatory bowel diseases with time, based on systematic review. Gastroenterology 2012;142:46-54. e42.

2. Ng WK, Wong SH, Ng SC. Changing epidemiological trends of inflammatory bowel disease in Asia. Intest Res 2016;14:111-119.

3. Loftus EV Jr. Clinical epidemiology of inflammatory bowel disease: incidence, prevalence, and environmental influences. Gastroenterology 2004;126:1504-1517.

4. Yoon SM. Micronutrient deficiencies in inflammatory bowel disease: trivial or crucial? Intest Res 2016;14:109-110.

5. Huang S, Ma J, Zhu M, Ran Z. Status of serum vitamin B(12) and folate in patients with inflammatory bowel disease in China. Intest Res 2017;15:103-108.

6. Shin IS, Seok H, Eun YH, et al. Wernicke's encephalopathy after total parenteral nutrition in patients with Crohn's disease. Intest Res 2016;14:191-196.

7. Sadeghian M, Saneei P, Siassi F, Esmaillzadeh A. Vitamin D status in relation to Crohn's disease: meta-analysis of observational studies. Nutrition 2016;32:505-514.

8. Siffledeen JS, Siminoski K, Steinhart H, Greenberg G, Fedorak RN. The frequency of vitamin D deficiency in adults with Crohn's disease. Can J Gastroenterol 2003;17:473-478.

9. Ulitsky A, Ananthakrishnan AN, Naik A, et al. Vitamin D deficiency in patients with inflammatory bowel disease: association with disease activity and quality of life. JPEN J Parenter Enteral Nutr 2011;35:308-316.
10. Mouli VP, Ananthakrishnan AN. Review article: vitamin D and inflammatory bowel diseases. Aliment Pharmacol Ther 2014; 39:125-136.

11. Garg M, Lubel JS, Sparrow MP, Holt SG, Gibson PR. Review article: vitamin D and inflammatory bowel disease: established concepts and future directions. Aliment Pharmacol Ther 2012; 36:324-344.

12. O'Sullivan M. Vitamin D as a novel therapy in inflammatory bowel disease: new hope or false dawn? Proc Nutr Soc 2015;74:5-12.

13. Hart AL. Vitamin D and inflammatory bowel disease: chicken or egg? Inflamm Bowel Dis 2013;19:459-460.

14. Theodoratou E, Tzoulaki I, Zgaga L, Ioannidis JP. Vitamin D and multiple health outcomes: umbrella review of systematic reviews and meta-analyses of observational studies and randomised trials. BMJ 2014;348:g2035.

15. Ye BD, Jang BI, Jeen YT, et al. Diagnostic guideline of Crohn's disease. Korean J Gastroenterol 2009;53:161-176.

16. Choi CH, Jung SA, Lee BI, et al. Diagnostic guideline of ulcerative colitis. Korean J Gastroenterol 2009;53:145-160.

17. Vermeire S, Schreiber S, Sandborn WJ, Dubois C, Rutgeerts P. Correlation between the Crohn's disease activity and HarveyBradshaw indices in assessing Crohn's disease severity. Clin Gastroenterol Hepatol 2010;8:357-363.

18. Walsh AJ, Ghosh A, Brain AO, et al. Comparing disease activity indices in ulcerative colitis. J Crohns Colitis 2014;8:318-325.

19. Travis SP, Higgins PD, Orchard T, et al. Review article: defining remission in ulcerative colitis. Aliment Pharmacol Ther 2011; 34:113-124.

20. Best WR. Predicting the Crohn's disease activity index from the Harvey-Bradshaw Index. Inflamm Bowel Dis 2006;12:304-310.

21. Harvey RF, Bradshaw JM. A simple index of Crohn's-disease activity. Lancet 1980;1:514.

22. Rosen CJ. Clinical practice: vitamin D insufficiency. N Engl J Med 2011;364:248-254.

23. Torki M, Gholamrezaei A, Mirbagher L, Danesh M, Kheiri S, Emami MH. Vitamin D deficiency associated with disease activity in patients with inflammatory bowel diseases. Dig Dis Sci 2015;60: 3085-3091.

24. Han YM, Yoon H, Lim S, et al. Risk factors for vitamin D, zinc, and selenium deficiencies in Korean patients with inflammatory bowel disease. Gut Liver 2017;11:363-369.

25. Fu YT, Chatur N, Cheong-Lee C, Salh B. Hypovitaminosis D in adults with inflammatory bowel disease: potential role of ethnicity. Dig Dis Sci 2012;57:2144-2148.

26. McCarthy D, Duggan P, O'Brien M, et al. Seasonality of vitamin D status and bone turnover in patients with Crohn's disease. Aliment Pharmacol Ther 2005;21:1073-1083.

27. Tajika M, Matsuura A, Nakamura T, et al. Risk factors for vitamin 
D deficiency in patients with Crohn's disease. J Gastroenterol 2004;39:527-533.

28. Leichtmann GA, Bengoa JM, Bolt MJ, Sitrin MD. Intestinal absorption of cholecalciferol and 25-hydroxycholecalciferol in patients with both Crohn's disease and intestinal resection. Am J Clin Nutr 1991;54:548-552.

29. Kabbani TA, Koutroubakis IE, Schoen RE, et al. Association of vitamin $\mathrm{D}$ level with clinical status in inflammatory bowel disease: a 5-year longitudinal study. Am J Gastroenterol 2016;111:712719.

30. Garg M, Rosella O, Lubel JS, Gibson PR. Association of circulating vitamin D concentrations with intestinal but not systemic inflammation in inflammatory bowel disease. Inflamm Bowel Dis 2013;19:2634-2643.

31. Kelly P, Suibhne TN, O'Morain C, O'Sullivan M. Vitamin D status and cytokine levels in patients with Crohn's disease. Int J Vitam
Nutr Res 2011;81:205-210.

32. Blanck S, Aberra F. Vitamin d deficiency is associated with ulcerative colitis disease activity. Dig Dis Sci 2013;58:1698-1702.

33. Dumitrescu G, Mihai C, Dranga M, Prelipcean CC. Serum 25hydroxyvitamin D concentration and inflammatory bowel disease characteristics in Romania. World J Gastroenterol 2014;20: 2392-2396.

34. Frigstad SO, Høivik M, Jahnsen J, et al. Vitamin D deficiency in inflammatory bowel disease: prevalence and predictors in a Norwegian outpatient population. Scand J Gastroenterol 2017;52: 100-106.

35. Raftery T, Merrick M, Healy M, et al. Vitamin D status is associated with intestinal inflammation as measured by fecal calprotectin in Crohn's disease in clinical remission. Dig Dis Sci 2015; 60:2427-2435. 